\title{
Rationalizing Treatment for Gynecological Cancers During the COVID- 19 Pandemic: An Indian Experience
}

\author{
G. Y. Srinivasa ${ }^{1}\left([) \cdot\right.$ Treshita Dey $^{1}\left(\mathbb{D} \cdot\right.$ Vanita Suri $^{2} \cdot$ Rashmi Bagga $^{2} \cdot$ Vanita Jain $^{2} \cdot$ Sandeep Gadela $^{1} \cdot$

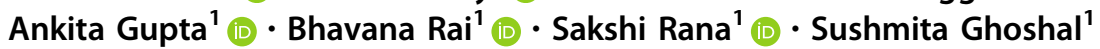

Received: 25 May 2020 / Revised: 27 July 2020 / Accepted: 17 August 2020/Published online: 31 August 2020

(C) Association of Gynecologic Oncologists of India 2020

\begin{abstract}
Purpose The entire world is currently facing a devastating crisis due to growing coronavirus pandemic, which was declared as a public health emergency by the World Health Organization on March 11, 2020. Management of cancer patients at this time is an overwhelming task. This study highlights our experience in the management of patients of gynecological malignancies over a period of 2 months during the COVID-19 pandemic.

Methods Patients of confirmed gynecological malignancies who visited our outpatient clinic and those who received radiotherapy/chemotherapy in March and April 2020 were included for analysis. Guidelines issued by the National Institute of Health and Care Excellence, National Health Service, MD Anderson Cancer Centre and those by young oncologists in Italy were followed with minor modifications while managing the logistics and health worker safety.

Results A total of 160 patients were treated in our department during this time period. In total, $44.4 \%$ of patients on treatment had associated comorbidities that imposed an additional risk. One hundred twenty-three patients continued treatment with their initial plan of radiotherapy or chemotherapy. New patients were prioritized based on the severity of clinical symptoms and whether the expected outcome would significantly affect their survival and quality of life. Patients were monitored for the development of treatment-related toxicities and COVID-19-related symptoms.

Conclusions All oncology personnel need to identify the correct balance between risks and benefit and then proceed with further management. Thus, it is essential to cautiously select patients for treatment, minimizing the risk of exposure but adequately addressing the underlying disease.
\end{abstract}

Keywords COVID-19 · Gynecological malignancies · Radiotherapy $\cdot$ Chemotherapy $\cdot$ Brachytherapy

\section{Introduction}

The entire world is currently facing a devastating crisis due to growing coronavirus (COVID19) pandemic, which was declared as a public health emergency by the World Health Organization on March 11, 2020. The USA, Spain and Italy have been the worst affected nations to date [1]. The

Bhavana Rai

bhavana1035@gmail.com

1 Department of Radiotherapy and Oncology, Post Graduate Institute of Medical Education and Research, Chandigarh 160012, India

2 Department of Obstetrics and Gynecology, Post Graduate Institute of Medical Education and Research, Chandigarh, India situation is no better in India, over 6.25 lakh confirmed cases with 18.2 thousand deaths [2].

Various strategies have been implemented to control the spread of this disease, and this has tremendously affected the healthcare workers who are working on the frontline to overcome this tragic infection. Available data suggest that the case fatality rate increases in the presence of high-risk features like age $\geq 65$ years, cardiovascular diseases, diabetes, hypertension, chronic lung disease, immunosuppression including cancer, chronic kidney disease and obesity (Body Mass Index $\geq 40$ ) [3]. Management of cancer patients at this time is an overwhelming task since these patients are inherently immunocompromised either due to the disease process itself or due to associated therapy, which is often complicated by the presence of comorbidities. 
European Society of Medical Oncology has developed a three-tiered approach to guide management of patients during the COVID-19 pandemic based on the Cancer Care Ontario criteria and the Magnitude of Clinical Benefit Scale, incorporating value-based prioritization [4]. Most of the gynecological malignancies as per this risk stratification fall under high- and medium-priority groups.

It is important to decide whether to continue treatment or withhold the same since it may impact the disease outcome and survival. It is hence imperative to continue treatment for high-priority patients, choose the treatment options wisely for the medium-priority patients according to the risk-benefit ratio and safely delay treatment for the low-priority ones. But, in LMIC like India, cancer patients are affected the most due to the COVID-19 pandemic that has hindered their management and affected the disease outcomes. Hereby, we highlight our experience in the management of patients of gynecological malignancies with limited resources over a period of 2 months during the COVID-19 pandemic that may help others in categorizing the treatment in this crucial time.

\section{Methods}

This analysis was conducted in the Department of Radiotherapy and Oncology, Post Graduate Institute of Medical Education and Research, Chandigarh, a tertiary-care institute which also caters to patients referred from multiple states in North India. Patients of confirmed gynecological malignancies who visited our outpatient clinic and those who received radiotherapy/chemotherapy in March and April 2020 were included for analysis.

Guidelines issued by the National Institute of Health and Care Excellence, National Health Service, MD Anderson Cancer Centre and those by young oncologists in Italy were followed while managing the logistics and health worker safety [5-8]. No routine follow-ups were encouraged. A COVID checklist was prepared and entered for every patient beforehand. Patients having positive international travel history or contact history with a known positive case, those with history of recent hospital stay, fever, anorexia, sore throat, cough, sputum production, shortness of breath, anosmia, diarrhea or conjunctivitis or any symptoms suggestive of suspected COVID-19 were sent for screening and further testing. Being a tertiary-care center, we have a dedicated 250-bedded COVID hospital where all confirmed cases of COVID-19 throughout the city were admitted, and this hindered our inpatient service.

\section{General Measures}

For patients:
1. Patients and attendants were educated about hand hygiene, social distancing, use of masks and symptoms that would require urgent medical care.

2. Accompanying family members were limited to only one person.

3. Crowded places were avoided.

4. Initially, clinical history and thermal screening were deemed adequate before performing any invasive procedure. However, later, due to change in the scenario of pandemic and our institutional protocol, all patients undergoing invasive procedures were tested for COVID-19 by reverse transcriptase-polymerase chain reaction (RT-PCR).

For healthcare workers:

1. All workers were screened every day at the time of reporting for duty for any respiratory or flu-like symptoms, any contact or travel history and thermal screening.

2. All healthcare workers were trained about proper handwashing, social distancing, use of protective gear including masks and were provided with the same as per the guidelines of Ministry of Health and Family Welfare, India [9].

3. Those healthcare workers involved in performing any invasive procedure in every department and those working in the dedicated COVID hospital had to wear the complete three-layer personal protective equipment (PPE) kit.

4. Prophylactic hydroxychloroquine (HCQ) was given as an optional treatment for healthcare workers.

5. All healthcare workers posted for COVID duty were tested 1 week after the completion of their posting. For those with accidental exposure with a confirmed case, the tests were done immediately with a quarantine period of 14 days.

6. One-third of the total staff were posted in every department for 14 days, followed by quarantine period of 14 days.

Outpatient department Visits:

1. As an institutional protocol, patients requiring emergency management and those in whom treatment delays were expected to be associated with a dismal prognosis were registered on a priority basis initially.

2. Outpatient department visits are restricted only to patients with an absolute necessity to address an acute oncologic issue and those under active treatment for their disease.

3. The number of physicians and healthcare providers was limited.

4. Telemedicine facilities: All patients were encouraged teleconsultation on the phone numbers, which were 
widely publicized on the institute Web sites at any time between $8 \mathrm{AM}$ and $1 \mathrm{PM}$. The available details including investigations were thoroughly studied, and all their complaints were addressed within the same day. Those on long-term follow-up with no clinical symptoms were asked not to visit the OPD. All new cases were given appointments to visit OPD with all the necessary investigations.

Workup of patients:

1. The initial biopsy of all patients was done in the Department of Gynecology.

2. Complete staging workup was done on OPD basis depending on the site and histology.

3. Further management was decided by the DMG (disease management group) over virtual meetings, depending on the stage and general condition of the patient.

\section{Surgery}

All patients requiring surgery were assessed critically by at least two consultants of the Unit to confirm the decision for surgery. Alternate nonsurgical/conservative managements were explored before surgical treatment. Surgeries were restricted to urgent oncology or emergency cases.

Choice of anesthesia Regional anesthesia was preferable to avoid the aerosol-generating events of intubation and extubation. It was discussed with anesthesiology colleagues.

Route of surgery Considerations regarding the choice of surgical route include patient comorbidities (such as but not limited to obesity, diabetes, cardiovascular disease). Minimally invasive surgery procedures were chosen for short surgical procedures to minimize the risk to healthcare workers.

Surgical technique was modified to minimize aerosol generation and duration of surgery. The frozen section should be avoided as far as possible. It was done only if it is likely to change the intra-op management. Institutional guidelines for the use of PPE are mentioned in Table 1.

Postoperative stay

It was minimized

COVID test was repeated if the stay was $>2$ weeks

Patients were advised to come for follow-up by appointment via teleconsultation

Radiotherapy treatment

1. All technologists handled the patients wearing disposable masks, gloves and face shields and were made to wash their hands after treating each patient with soap and water followed by application of alcohol-based hand sanitizers.

2. After treatment of each patient, the couch was cleaned with alcohol-based disinfectants.

3. Palliative hypofractionated radiotherapy was converted to single-fraction radiotherapy.

\section{Brachytherapy}

1. Every effort was made to minimize the total length of stay in hospital (two fractions of 9 Gy high dose rate preferred over four fractions of 7 Gy each)

2. Procedures were preferably done under conscious sedation, and only the difficult cases were done under general anesthesia to minimize exposure by avoiding the risks related to endotracheal intubation.

3. For postoperative adjuvant brachytherapy, the riskbenefit ratio was discussed with the patient while taking into account the clinical condition and local epidemiological context.

Chemotherapy

1. Risk and benefit regarding the continuation of therapy were explained on a one-to-one basis.

2. Routine use of filgrastim was mandated to reduce the incidence of neutropenia in patients receiving combination therapy.

Table 1 Guidelines for the use of PPE

\begin{tabular}{|c|c|c|c|c|c|}
\hline & Gloves & $\begin{array}{l}\text { Impervious } \\
\text { bodysuit }\end{array}$ & Mask & Vision/hood & $\begin{array}{l}\text { Linen } \\
\text { gown }\end{array}$ \\
\hline \multicolumn{6}{|l|}{ Suspected or confirmed COVID-19 cases } \\
\hline Operating theaters & Yes & Yes & N 95 & Yes & $\begin{array}{l}\text { Yes, } \\
\text { sterile }\end{array}$ \\
\hline \multicolumn{6}{|l|}{ COVID negative case or report awaited } \\
\hline Operating theaters & Yes & No & $\begin{array}{l}\text { Surgical/N95 when COVID test not } \\
\text { available }\end{array}$ & Yes & $\begin{array}{l}\text { Yes, } \\
\text { sterile }\end{array}$ \\
\hline $\begin{array}{l}\text { Minor OT, USG guided and FNAC } \\
\text { procedures }\end{array}$ & Yes & No & Filtering face piece 1 (FFP1) & Yes & Yes \\
\hline
\end{tabular}


3. The total number of patients receiving chemotherapy was capped depending on the total number of available beds in our daycare ward.

4. Weekly regimens were avoided

\section{Statistical Analysis}

Statistical analysis was done using the Statistical Program for Social Sciences (SPSS v 23, IBM Corp, USA) software. Descriptive analysis was done for the patient demographics, disease and treatment characteristics. Summary of statistics, including frequency and mean as applicable, was obtained.

\section{Results}

A total of 160 patients were treated in our department during this time period. Patient characteristics and their treatment regimens are detailed in Tables 1, 2 and 3. In total, $44.4 \%$ of patients on treatment had associated comorbidities that imposed an additional risk. One hundred twenty-three patients continued treatment with their initial plan of radiotherapy or chemotherapy.

New patients who were referred to our department were prioritized based on the severity of clinical symptoms and whether the expected outcome from treatment would significantly affect their survival and quality of life. Thirtyone new patients were registered during this study period, including 12 patients of carcinoma cervix, three patients of carcinoma endometrium and 16 of carcinoma ovary.

\section{Cervical Cancer}

Stage distribution is illustrated in Table 2. No cases of stage IA were reported in this period. All the seven cases of stage IB were discussed in DMG and considered for definitive chemoradiation [CRT].

Of the total 74 patients of carcinoma cervix, 62 were on treatment (34 on radical CRT, two on palliative radiotherapy, 23 due for brachytherapy and three on palliative chemotherapy). Remaining 12 patients were newly registered, and ten such cases were started on radical CRT, one on adjuvant radiotherapy and other on palliative chemotherapy. All patients on radical CRT underwent planning computed tomography (CT) of abdomen and pelvis before starting radiation and were treated with $3 \mathrm{D}$ conformal RT. No treatment interruption was encouraged for patients undergoing radical radiation in order to maximize local disease control and survival. Two patients received palliative $8 \mathrm{~Gy}$ single fraction to the pelvis for
Table 2 Patient and disease characteristics

\begin{tabular}{|c|c|}
\hline Characteristics & Frequency \\
\hline Mean age (years) & 51.16 \\
\hline \multicolumn{2}{|l|}{ Comorbidity } \\
\hline Diabetes mellitus & $10(6.3 \%)$ \\
\hline Diabetes and hypertension & $10(6.3 \%)$ \\
\hline Hypertension & $33(20.6 \%)$ \\
\hline Hypothyroidism & $11(6.9 \%)$ \\
\hline Others & $7(4.4 \%)$ \\
\hline None & $89(55.6 \%)$ \\
\hline \multicolumn{2}{|l|}{ Diagnosis } \\
\hline \multicolumn{2}{|l|}{ Carcinoma cervix $(n=74)$} \\
\hline Squamous cell ca & $72(97.3 \%)$ \\
\hline Adenocarcinoma & $2(2.7 \%)$ \\
\hline \multicolumn{2}{|c|}{ Carcinoma endometrium $(n=17)$} \\
\hline Endometrioid & $10(58.8 \%)$ \\
\hline High-grade serous & $1(5.9 \%)$ \\
\hline Carcinosarcoma & $6(35.3 \%)$ \\
\hline \multicolumn{2}{|l|}{ Carcinoma ovary $(n=67)$} \\
\hline High-grade serous & $53(79.1 \%)$ \\
\hline Mucinous & $5(7.5 \%)$ \\
\hline Low-grade serous & $3(4.5 \%)$ \\
\hline Germ cell tumor & $5(7.5 \%)$ \\
\hline Endometrioid & $1(1.4 \%)$ \\
\hline \multicolumn{2}{|l|}{ Carcinoma vault $(n=2)$} \\
\hline Squamous cell ca & $2(100 \%)$ \\
\hline \multicolumn{2}{|l|}{ Stage } \\
\hline \multicolumn{2}{|l|}{ Carcinoma cervix } \\
\hline Early stage & $7(9.5 \%)$ \\
\hline Locally advanced & $65(87.8 \%)$ \\
\hline Distant metastasis & $2(2.7 \%)$ \\
\hline \multicolumn{2}{|l|}{ Carcinoma endometrium } \\
\hline Early stage & $10(58.9 \%)$ \\
\hline Locally advanced & $7(41.1 \%)$ \\
\hline \multicolumn{2}{|l|}{ Carcinoma ovary } \\
\hline Early stage & $5(7.5 \%)$ \\
\hline Locally advanced & $48(71.6 \%)$ \\
\hline Distant metastasis & $14(20.9 \%)$ \\
\hline \multicolumn{2}{|l|}{ Carcinoma vault } \\
\hline Stage II & $2(100 \%)$ \\
\hline \multicolumn{2}{|l|}{ Number of visits } \\
\hline 1 & $77(48.1 \%)$ \\
\hline 2 & $81(50.6 \%)$ \\
\hline 3 & $2(1.3 \%)$ \\
\hline
\end{tabular}

symptom control because of extensive locoregional disease.

We modified our institutional protocol during this period which allowed us to categorize patients depending on the 
Table 3 Radiotherapy characteristics

\begin{tabular}{lllll}
\hline Diagnosis & Type & Intent & Patients who continued treatment & New patients \\
\hline Ca cervix & EBRT $^{\mathrm{a}}$ & Radical & $29(39.2 \%)$ & $10(13.5 \%)$ \\
& & Supplementary & $5(6.8 \%)$ & 0 \\
& & Adjuvant & 0 & $1(1.4 \%)$ \\
& & Palliative & $2(2.7 \%)$ & 0 \\
Ca endometrium & ICBT $^{\mathrm{b}}$ & $23(31.1 \%)$ & & $1(5.9 \%)$ \\
& EBRT $^{\text {Ca vault }}$ & Adjuvant & $5(29.4 \%)$ & \\
\hline
\end{tabular}

${ }^{\mathrm{a}}$ External beam radiation

${ }^{\mathrm{b}}$ Intracavitary brachytherapy

${ }^{\mathrm{c}}$ Vault brachytherapy

disease response to initial CRT and its probable survival outcome. A total of 34 patients were assessed for brachytherapy. Five patients received pelvic boost by external radiation due to heavy parametrial burden and inadequate clinical response to initial CRT and nonavailability of in-patient services restricting interstitial brachytherapy. Remaining 23 patients underwent intracavitary brachytherapy. The dose schedule was customized depending on the disease burden during brachytherapy. Twenty patients received 9 Gy HDR for two fractions, 1 week apart, and three patients received 7 Gy for four fractions and two fractions per day [6-h gap between fraction], 1 week apart. If the patients had suitable anatomy, intracavitary brachytherapy was done on OPD basis under conscious sedation, and all other cases were shifted to the operation theater for general anesthesia. There were two patients of carcinoma vault, and they continued with two sessions of intravaginal brachytherapy, weekly 6 Gy each following pelvic CRT. Details of radiotherapy are mentioned in Table 3 .

All patients on CRT received concurrent chemotherapy with weekly cisplatin without any treatment interruption. Only four patients received palliative chemotherapy with paclitaxel and carboplatin after thoroughly explaining the risk-benefit ratio.

\section{Ovarian Cancer}

Stage distribution is illustrated in Table 2.

Forty-five patients carried on with their planned treatment (15 on neoadjuvant, 15 on adjuvant, seven on secondline and eight on third-line chemotherapy). Sixteen new symptomatic cases of locally advanced ovarian cancer were registered and were started on neoadjuvant chemotherapy. Both unresectable and resectable cases spread beyond the pelvis were planned for neoadjuvant chemotherapy after discussing in DMG. All patients were started on three-weekly chemotherapy regimen. Weekly chemotherapy was discouraged due to the risk of exposure on repeated visits; instead, $20-25 \%$ dose reduction was made in the three weekly schedule in elderly patients with comorbidities, to reduce the rates of toxicities as an institutional protocol. Those started on neoadjuvant chemotherapy were discussed in DMG after completion of three cycles, and it was decided to complete six cycles followed by an assessment for surgery. Six patients with radiological and biochemical platinum-sensitive relapses were started second- or third-line chemotherapy regimen.

No treatment-related acute toxicity or mortality was observed during this time period. No treatment interruption was allowed for those treated with radical intent. For all patients on neoadjuvant therapy, the number of chemotherapy cycles was increased up to six to delay the interval surgery and its complications. This plan was incorporated after discussion in the gynecological disease management group. Details of chemotherapy are mentioned in Table 4

\section{Endometrial Cancer}

Stage distribution is illustrated in Table 2.

Fourteen patients carried on with their planned treatment (four on adjuvant, two on neoadjuvant and three on palliative chemotherapy, five on pelvic radiation), and the remaining three were newly registered patients. Two were high-intermediate-risk stage IB patient, started on vault brachytherapy, 7 Gy each for three fractions, on alternate days to complete the entire course of radiation within a week. Another one was a stage III patient referred for adjuvant therapy, initiated on radical pelvic radiation, and adjuvant chemotherapy was delayed. All radically treated patients did not have any treatment interruption. 
Table 4 Chemotherapy characteristics

\begin{tabular}{llcc}
\hline Diagnosis & Intent & Patients who continued planned chemotherapy & Newly started on chemotherapy \\
\hline Ca cervix & Concurrent & $29(39.2 \%)$ & $10(13.5 \%)$ \\
& Palliative & $3(4.1 \%)$ & $1(1.4 \%)$ \\
Ca endometrium & Neoadjuvant & $2(11.8 \%)$ & 0 \\
& Adjuvant & $4(23.5 \%)$ & 0 \\
Palliative & $3(17.6 \%)$ & 0 \\
Ca ovary & Neoadjuvant & $15(22.4 \%)$ & $13(19.4 \%)$ \\
& Adjuvant & $15(22.4 \%)$ & $3(4.6 \%)$ \\
& Second line & $7(10.5 \%)$ & $4(5.9 \%)$ \\
\end{tabular}

\section{Follow-Up}

At first follow-up, patients were asked to visit the OPD as per the given appointment for response assessment. Those having a complete response were given the option of further follow-up on teleconsultation. Further management of patients having residual disease was decided depending upon the response and clinical status.

All patients enrolled in this study were contacted over telephone at the end of the study period to enquire about the presence of fever, cough, shortness of breath, sputum production, etc. Patients were monitored for the development of treatment-related toxicities, e.g., nausea, vomiting, diarrhea, anorexia, peripheral neuropathy, bladder symptoms, etc. A brief account was also kept on whether the patients have visited any hotspot zone or came in contact with a known coronavirus positive case. None of our patients developed any suspicious symptoms, nor was there any treatment-related mortality or morbidity.

\section{Discussion}

In LMIC like India, management of cancer amidst the COVID-19 crisis has been severely affected. Both the diagnosis and the management of these patients have been delayed, which has increased the burden on the health facilities. Moreover, there are no national guidelines that highlight these critical issues. In limited-resource settings, it becomes challenging to manage the scenario. Hence, in this study, we have highlighted our institutional experience in treating oncology patients during the COVID-19 pandemic that may pave the way for future references.

During the COVID-19 outbreak, we have actively managed 160 cases of gynecological malignancies. Of these, 123 patients carried on with their planned management with radiotherapy or chemotherapy or both. Ten new cases of locally advanced cervical cancer were started on radical CRT. For endometrial carcinoma, three new patients were registered, one locally advanced carcinoma endometrium on adjuvant pelvic radiation and others stage IB high intermediate risk on vault brachytherapy. A total of 16 new cases of carcinoma ovary were initiated on chemotherapy, nine cases of stage III high-grade serous ovarian cancer started on adjuvant chemotherapy with three-weekly paclitaxel and carboplatin regimen and the rest underwent neoadjuvant chemotherapy based on their respective histology.

It is clear that all cancer patients are at high risk of developing severe illness, but delaying treatment can hamper the prognosis. Thus, it is essential to cautiously select patients for treatment, minimizing the risk of exposure but adequately addressing the underlying disease. Keeping in mind the priority groups suggested by various international groups, we see that majority of gynecological malignancies fall under high- and medium-priority groups. Hence, postponing treatment in most of these does not act in favor of the patient.

The number of visits to the hospital has been minimized for each patient, thus reducing the risk of possible exposure. About $48 \%$ of patients visited only once, while $50.6 \%$ visited twice during this time period. Due to cautious screening protocols followed in our department, we have not encountered any treatment-related morbidity or mortality and COVID-19-related symptoms so far.

Carcinoma cervix being a potentially curable malignancy in locally advanced stages falls under the high-priority group. "Overall treatment time determines local control along with concurrent chemotherapy [9]. Treatment time over 8 weeks was associated with detrimental pelvic control with control rate falling by $0.85 \%$ per day across all stages [10]. It has been extensively studied that the addition of brachytherapy led to a survival benefit in such patients" [11]. Hence, all locally advanced cervical cancer patients 
should receive upfront radical CRT along with brachytherapy. Neither hypofractionation nor neoadjuvant chemotherapy should be used, given their detrimental impact on outcome [12]. According to the American Brachytherapy Society guidelines, brachytherapy procedures for cervical cancer patients should not be delayed in patients without COVID-19 symptoms [13]. At our center, all patients of cervical cancer continued with radical treatment without any intentional delay. Interstitial brachytherapy could not be administered due to nonavailability of inpatient services. Hence, those with heavy parametrial disease or those with a poor clinical response to the first phase of radiation were planned for pelvic boost by external radiation. Palliative radiotherapy was given as a single fraction of radiation to achieve adequate symptom control and minimize the exposure.

In carcinoma ovary, a retrospective study by Starbuck et al. [14] has shown that timely chemotherapy completion was associated with better survival and high complete response rates. It was seen by Williams et al. that mortality from chemotherapy was doubled in the presence of COVID-19 infection, in the age group of over 50 years [15]. In patients who were initiated on neoadjuvant chemotherapy, plans were modified to complete chemotherapy to six cycles instead of the usual three before assessment for interval debulking surgery. This plan was incorporated after discussion in the gynecological disease management group and the institutional decision of suspending elective cases for surgery as we are a designated COVID hospital. Continuation or initiation of second-line and third-line chemotherapy was done only after a thorough discussion regarding the potential benefits and risks with both the patient and her caregivers.

In carcinoma endometrium, large retrospective analysis has suggested that prolonged time between surgery and adjuvant therapy is associated with poor survival even in early-stage disease [16]. For adjuvant brachytherapy in carcinoma endometrium, American Brachytherapy Society suggested consented delays till 12 weeks after surgery, since with further delay, survival may be compromised [13]. In our study population, $35.3 \%$ of the patients were of stage I and $23.5 \%$ of stage II. Hence, these patients carried out their treatment without any interruptions. Also, the locally advanced cases requiring both adjuvant chemotherapy and radiotherapy were started on radiation first in view of decreased risk of compromised immunity. Vault brachytherapy, as indicated, was continued without any interruption.

These guidelines were followed based on the institute's experience and were tailored for every patient. No national guidelines have yet been instituted. However, since no morbidity occurred during this time period, we plan to continue using the same principles until the resolution of the crisis.

\section{Strategies in Treating Gynecological Oncology Cases During the Pandemic}

Strategies for managing OPD and limiting the number of patients

1. The need for hospital visits should be prioritized by giving teleconsultation services.

2. Teleconsultation for follow-up should be encouraged. Those patients requiring urgent medical attention or those with a scope of complete cure should be given priority.

3. The number of patients attending OPD, including new case registration, should be limited to avoid unnecessary crowding. Patients should be encouraged for teleconsultation prior to their OPD visits which will allow us to prioritize and give appointments as necessary.

4. Emphasis should be given for developing a comprehensive network with $\mathrm{CHC}$ and PHC for minimum basic care for cancer patients and minimizing referrals

Strategies for treatment

1. Early-stage and operable patients should be taken for surgery. All patients requiring surgery should be assessed critically by at least two consultants to confirm the decision for surgery. Alternate nonsurgical (radical radiation) managements should be explored before surgical treatment.

2. Modification of curative radiotherapy fractionation schedules should be made to reduce the number of hospital visits.

3. There should be capping of the number of patients on each radiotherapy machine to avoid overcrowding and minimize exposure

4. Weekly chemotherapy regimen should be avoided

5. There should be no hindrance in continuing palliative care services including the use of morphine

6. Increased use of single-fraction hypofractionated palliative radiotherapy should be encouraged with the advantage of minimal hospital stay and patient convenience. This would also create more available slots for patients requiring radical radiotherapy.

\section{Conclusion}

Considering the worsening global scenario of the COVID19 pandemic, all oncology personnel need to identify the correct balance between risks and benefit and then proceed 
with further management. Thus, maintaining all necessary precautions, treatment can be continued for a suitable subset of cancer patients with minor modifications according to the availability of resources.

Acknowledgements We are grateful to Prof. Vanita Suri, Prof. Vanita Jain and Prof. Rashmi Bagga from the Department of Obstetrics and Gynecology for their contribution toward this manuscript.

Funding Not applicable.

Availability of Data and Material Data are available whenever needed.

\section{Compliance with Ethical Standards}

Conflict of interest The authors declare that they have no conflict of interests.

\section{References}

1. COVID-19 situation reports [Internet]. Who.int. 2020 [cited 25 April 2020]. https://www.who.int/emergencies/diseases/novelcoronavirus-2019/situation-reports.

2. MoHFW I Home [Internet]. Mohfw.gov.in. 2020 [cited 3 July 2020]. https://www.mohfw.gov.in/.

3. Coronavirus disease 2019 (COVID-19): are you at higher risk for severe illness? [Internet]. Cdc.gov.2020 [cited 25 April 2020]. https://www.cdc.gov/coronavirus/2019-ncov/specific-groups/ high-risk-complications.html.

4. Cancer Patient Management During the COVID-19 Pandemic [Internet]. Esmo.org.2020 [cited 20 April 2020]. https://www. esmo.org/guidelines/cancer-patient-management-during-thecovid-19-pandemic. .

5. Overview|COVID-19 rapid guideline: delivery of radiotherapylGuidancelNICE [Internet]. Nice.org.uk.2020 [cited 20 April 2020]. https://www.nice.org.uk/guidance/NG162.

6. [internet] England.nhs.uk.2020 [cited 23 April 20]. https://www. england.nhs.uk/coronavirus/wp-content/uploads/sites/52/2020/ 03/specialty-guide-acute-treatment-cancer-23-March-2020.pdf.
7. Ramirez PT, Chiva L, Eriksson A, Frumovitz M, Fagotti A, et al. COVID-19 global pandemic: options for management of gynecologic cancers. Int J Gynecol Cancer. 2020. https://doi.org/10. 1136/ijgc-2020-001419.

8. Lambertini M, Toss A, Passaro A, et al. Cancer care during the spread of coronavirus disease 2019 (COVID-19) in Italy: young oncologists' perspective. ESMO Open. 2020;5:e000759. https:// doi.org/10.1136/esmoopen-2020-000759.

9. National Guidelines for Infection Prevention and Control in Healthcare Facilities [Internet]. Mohfw.gov.in. 2020 [cited 30 April 2020]. https://www.mohfw.gov.in/pdf/National\%20Guide lines\%20for\%20IPC\%20in\%20HCF\%20-\%20final\%281\%29. pdf.

10. Perez CA, Grigsby PW, Castro-Vita H, Lockett MA. Carcinoma of the uterine cervix. I. Impact of prolongation of overall treatment time and timing of brachytherapy on outcome of radiation therapy. Int J Radiat Oncol Biol Phys. 1995;32(5):1275-88.

11. Han K, Milosevic M, Fyles A, et al. Trends in the utilization of brachytherapy in cervical cancer in the United States. Int J Radiat Oncol Biol Phys. 2013;87:111-9.

12. Gupta S, Maheshwari A, Parab P, et al. Neoadjuvant chemotherapy followed by radical surgery versus concomitant chemotherapy and radiotherapy in patients with stage IB2, IIA, or IIB squamous cervical cancer: a randomized controlled trial. J Clin Oncol. 2018;36:1548-55.

13. ABS Statement on Coronavirus [Internet]. American Brachytherapy Society. 2020 [cited 19 April 2020]. https://www. americanbrachytherapy.org/about-abs/abs-news/abs-statementon-coronavirus/.

14. Starbuck K, Szender J, Duncan W, Morrell K, et al. Prognostic impact of adjuvant chemotherapy treatment intensity for ovarian cancer. PLoS ONE. 2018;13(11):e0206913.

15. Williams M, Le Calvez K, Mi E, Chen J, Dadhania S, PakzadShahabi L. Estimating the Risks from COVID-19 infection in adult chemotherapy patients [Internet]. https://doi.org/10.33697/ ajur.2019.003.

16. Luo L, Shi W, Zhang Z. Tsai C 2017 Association of delayed adjuvant therapy and overall survival in early stage endometrial cancer. J Clin Oncol. 2017;35(15_suppl):5590.

Publisher's Note Springer Nature remains neutral with regard to jurisdictional claims in published maps and institutional affiliations. 